\title{
Repeated Exposure to Benzalkonium Chloride in the Ex Vivo Eye Irritation Test (EVEIT): Observation of Isolated Corneal Damage and Healing
}

\author{
Markus Frentz, 1,3 Miriam Goss, ${ }^{2}$ Martin Reim³ and Norbert F. Schrage1,2,3 \\ ${ }^{1}$ Aachen Centre of Technology Transfer in Ophthalmology, Aachen, Germany; 2Department of \\ Ophthalmology Cologne Merheim, Cologne, Germany; ${ }^{3}$ Department of Ophthalmology, Aachen University, \\ Aachen, Germany
}

\begin{abstract}
Summary - The prediction of side-effects is a key issue in the REACH initiative on chemicals, in the production of cosmetics and in the preclinical testing of drugs. A new ex vivo test for repeated substance application is presented, that is able to identify corrosive and irritant effects on the eye by using crucial endpoints, such as cellular and morphological damage, and healing characteristics. The test is intended to replace the Draize eye test and to improve the preclinical testing of drugs and chemicals that are likely to come into direct contact with the cornea. The Ex Vivo Eye Irritation Test (EVEIT) is a self-healing system, involving living corneas obtained from abattoir rabbit eyes. The corneas are cultured in a similar way to the method used during the transplantation of corneal grafts. The corneas are exposed to multiple small, mechanical abrasions, and then test substances are repeatedly dropped onto the centres of the corneas. The test substances applied in this study were citrate-buffered hyaluronate eye drops and an artificial tear replacement, with increasing concentrations of up to $0.1 \%$ benzalkonium chloride. A dose-dependent inhibition of recovery and impairment of the lactate production mechanism in the cornea was observed with benzalkonium chloride treatment.
\end{abstract}

Key words: benzalkonium chloride, cornea, ex vivo test, hyaluronic acid, replacement alternative, stroma.

Address for correspondence: Norbert Schrage, ACTO, Karlsburgweg 9, 52070 Aachen, Germany. E-mail: schrage@acto.de

\section{Introduction}

The Draize eye irritation test is used to evaluate the hazard from a single exposure of the eye to chemicals that result in lesions, and has been required by official guidelines for evaluation of biomedical devices and cosmetics (1). Up to now, it has been considered to be the gold standard in toxicological evaluations of the effects of chemicals on the eye, but it is criticised because it uses a scoring system based on subjective observations. The results of many in vivo studies have been collected in databases, including an ECETOC report (2).

The ideal 'modern' methodological approach is to avoid animal experiments altogether, which is currently best achieved by using the biomathematical models proposed by Prospisil et al. (3), but it has not yet been possible to replace the rabbit eye test with in vitro methods. A number of new approaches have been submitted to ECVAM and ICVAM for approval. Van Goethem et al. (4) have published details of a test involving corneal constructs as one promising possibility. However, such constructs do not reflect the mechanical structure and diffusion mechanisms of the living cornea. Furthermore, the transfer of endothelium to the model and its subsequent maintenance are still unresolved issues, since the outcome depends strongly on the origin of the tissues, the method of immortalisation, and the cyclic age of the cultures. The standardisation of culture conditions, serum supplies, and many other factors are also regarded as problematic issues, which may affect the predictive value of results (5).

Therefore, our goal was to develop a reliable irritation testing system, based on highly-standardised corneal cultures of the type routinely used during organ culture for subsequent transplantation. There is considerable knowledge of the metabolic equilibrium of such corneas within this culture system (6), and the rabbit eye is the most widely studied medical model in corneal research. We therefore standardised the culture method normally used for corneal organ culture, and developed determinants of irritation analysis within this new system, which are more objective than those that are currently used in the Draize model (1).

We were interested in demonstrating the reliability of our system, the Ex Vivo Eye Irritation Test (EVEIT), and in investigating whether benzalkonium chloride (BAC) could be identified as toxic, as determined by inhibition of epithelial recovery. We used repeated exposure to $\mathrm{BAC}$, with subsequent monitoring of wound healing and assessment of the metabolic status of the cornea. In addition, we 
addressed the key issues in efforts to reduce, refine and, finally, replace animal experiments (the Three $\mathrm{Rs}$ ), in order to establish the EVEIT as a reliable method for the prediction of relevant effects on the cornea in a short-term experimental set-up.

\section{Materials and Methods}

\section{The EVEIT}

The EVEIT uses only corneas that are freshlyobtained from the slaughter house, which involves a delay of up to eight hours prior to preparation for use. In the development of the method, the eyes were transported in complete heads cooled to $4^{\circ} \mathrm{C}$. The lids were briefly opened and Polyspectran ${ }^{\circledR}$ eye drops (Dr Thilo GmbH \& Co. KG, Freiburg, Germany) were applied into the conjunctival sac before transportation. Subsequently, the corneas were excised from the eyes with a $12 \mathrm{~mm}$ trephine, and the adhering tissues were gently removed. Each isolated cornea, with about $2 \mathrm{~mm}$ of adherent sclera, was placed in a special corneal culture chamber (ACTO e.V., Aachen, Germany), clamped between the upper and lower parts of the perfusion chamber. After the placement of the cornea, the chamber was gently filled with perfusion medium (Minimum Essential Medium [MEM], Cat. No. T031-05, Biochrom AG, Berlin, Germany), with added piperacillin $(2 \mathrm{mg} / \mathrm{ml}$; Ratiopharm $\mathrm{GmbH}$, Ulm, Germany), amikacin $(0.2 \mathrm{mg} / \mathrm{ml}$; Fresenius Kabi Deutschland GmbH, Bad Homburg von der Höhe, Germany), and nystatin (400U/ml; Valeant Pharmaceuticals Germany $\mathrm{GmbH}$, Eschborn, Germany). The medium was constantly replenished by using a micropumping system, with an entrance $\mathrm{pH}$ of $7.4 \pm$ 0.2 and a flow rate of $6 \mu \mathrm{l} / \mathrm{min}$. The chambers were maintained at $32^{\circ} \mathrm{C}$ in normal air and $100 \%$ relative humidity for 24 hours, prior to the use of the explanted corneas.

\section{Quality control}

After stabilisation for 24 hours in the EVEIT culture system, the epithelial integrity of the corneas was verified with fluorescein surface staining $(1.7 \mathrm{mg} / \mathrm{ml}$ fluorescein, sodium salt; Sigma-Aldrich, St. Louis, MO, USA), an assessment of the endothelial appearance from the back of the chamber, and the determination of lactate production, to ensure that the conditions for testing were standardised. If lactate production was lower than $1 \mathrm{mmol} / \mathrm{l}$, the endothelium showed obvious cell loss, or the epithelium exhibited fluorescein-positive regions, then the cornea concerned was excluded from further processing.

\section{Examination techniques}

The epithelial surface was examined microscopically. The surface was stained with fluorescein, and images were taken by using a Canon EOS 500 digital camera with a macro-lens (Canon, Inc., Tokyo, Japan). Samples of medium from the artificial anterior chamber outflow were taken continuously. After each hour (i.e. $360 \mu$ l total volume), the samples were analysed for glucose, lactate and $\mathrm{pH}$ (Bayer Automatic Analyser: Rapid Lab 860; Siemens Diagnostics, Fernwald, Germany).

\section{Mechanical abrasion}

A small dental drill (Arkansasschleifer 638XF, Meisinger, Neuss, Germany) was used to create four small abrasions on the cornea, each with an area of $0.3-0.6 \mathrm{~mm}^{2}$. All defects were stained daily with fluorescein, briefly flushed after three seconds with isotonic Ringer's solution (DeltaSelect $\mathrm{GmbH}$, Pfullingen, Germany) to remove excess fluorescein, and then photographed immediately. The yellow green fluorescence in Figure 1 indicates epithelial defects, initially due to mechanical abrasion, and, in the later stages of the experiment, due to the local toxicity of the applied substances.

\section{Application of test substances}

Unpreserved hyaluronate citrate drops (HYLOLASOP ${ }^{\circledR}$, Ursapharm Arzneimittel GmbH \& Co. KG, Saarbrücken, Germany) and an artificial tear replacement (a physiological saline solution containing $14.581 \mathrm{mmol} \mathrm{Ca} \mathrm{Ca}^{2+}$ in $0.9 \%$ saline solution; DeltaSelect $\mathrm{GmbH}$ ), with or without $0.001-0.1 \%$ BAC (w/v; Sigma-Aldrich Chemie GmbH, Munich, Germany), were applied by using two separate dropping devices, exactly over the apex of the cornea. A $20 \mu \mathrm{l}$ drop of each solution was applied hourly, with a maximum time difference of $30 \mathrm{~min}-$ utes between application of the test substances and the artificial tears. In terms of 'real-life' simulation, this test offers the opportunity to ascertain the effects of hourly applications of a drug, as would be utilised in a therapeutic regime. Higher frequencies of application would not be indicative of the 'reallife' clinical application of the drug. Eye drops were not applied simultaneously, on any occasion. A softtipped cannula, applying continuous suction, was placed on the lower side of the cornea in culture to remove excess fluid.

Negative controls were represented by two corneas, with four small abrasion sites on each, treated with MEM and artificial tear replacement 24 times per day (once per hour). Excess fluids were again removed by suction with a soft-tipped cannula, placed on the lower part of the anterior cornea. A further set of two intact corneas were used as control for vitality and integrity of the epithelium within the test system (i.e. no abrasions were made, and no test substances were applied). 


\section{Figure 1: A macroscopic photograph of a cornea within the EVEIT, with four corneal abrasions stained with fluorescein}

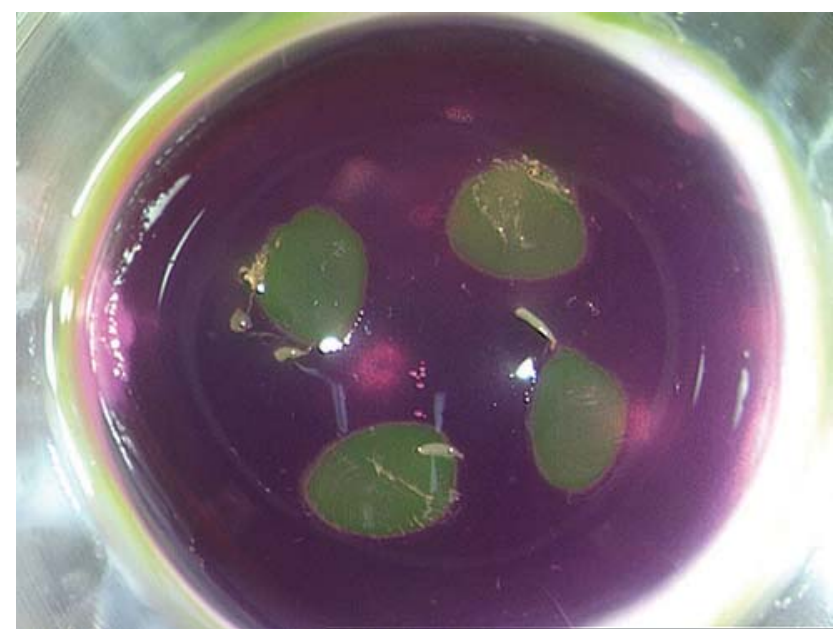

\section{Results}

The vitality controls, with no initial erosion, remained clear and showed no corneal opacity throughout the experimental period of four days (Figure 2). The negative controls treated with MEM showed considerable, but incomplete, healing (Figure 3); at day 4, corneal erosion was still evident, with a slight greyish appearance of the erosion and epithelial edges. Figure 4 shows corneas treated with hyaluronate citrate and artificial tears. The complete healing of the epithelial defects is clearly visible. A trace of fluorescein staining at the former centre of the epithelial defects was scarcely visible after four days.

Figures 5a-5e show corneas treated with hyaluronate citrate and artificial tears containing different concentrations of BAC. Dose-dependent swelling, with corneal stromal opacity, roughness of the epithelium, and disintegration of the epithelial surface at the highest concentrations, was observed. The acceleration of the damage to full necrosis, together with metabolic failure (as evidenced by the absence of lactate production), was clearly dependent on the BAC concentration (Figure 6).

Corneas treated with 24 drops per day (once per hour) of hyaluronate citrate and calcium-containing artificial tears with $0.001 \%$ BAC, showed damaged surface integrity, and no healing of the initial erosion was apparent (Figure 5a). The lactate concentration (Figure 6) remained in the expected range, showing that the tissue would still be able to recover and re-epithelialise, if the treatment with the toxic substance was stopped.

Increasing the concentration of $\mathrm{BAC}$ to $0.01 \%$ led to marked destruction of the epithelium, with extensive corneal erosion (Figure $5 \mathrm{~b}$ ). With $0.025 \%$ $\mathrm{BAC}$, the visible damage (Figure 5c) appeared almost the same as that which occurred with $0.01 \%$ BAC. However, lactate production by the $0.025 \%$ BAC-treated tissues was clearly impaired much earlier than it was with the lower concentration, decreasing to almost complete metabolic failure at

\section{Figure 2: A cornea within the EVEIT, serving as a vitality control}

a)

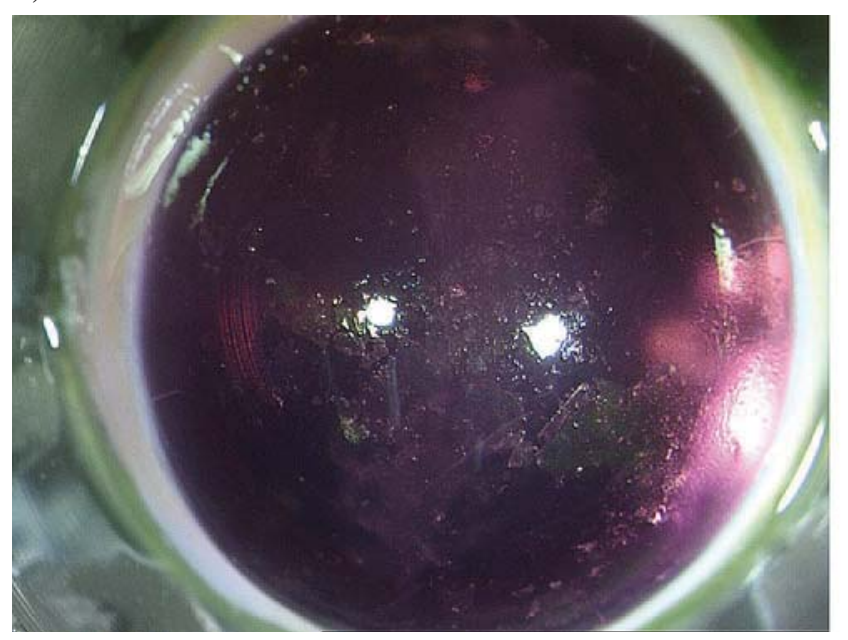

b)

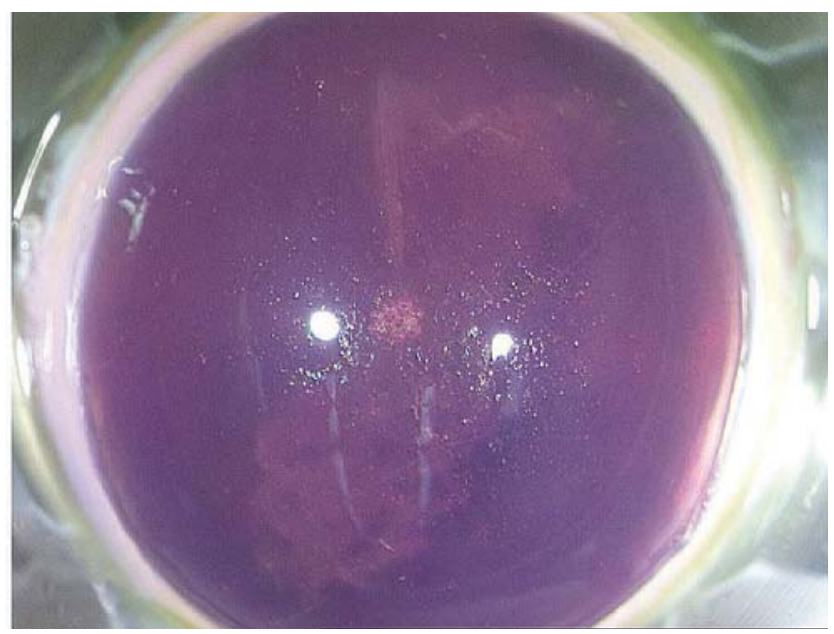

The images show the isolated cornea a) at the beginning (Day 0) and b) at the end (Day 4) of the experiment. 
Figure 3: A cornea within the EVEIT, treated with repeated drops of MEM

a)

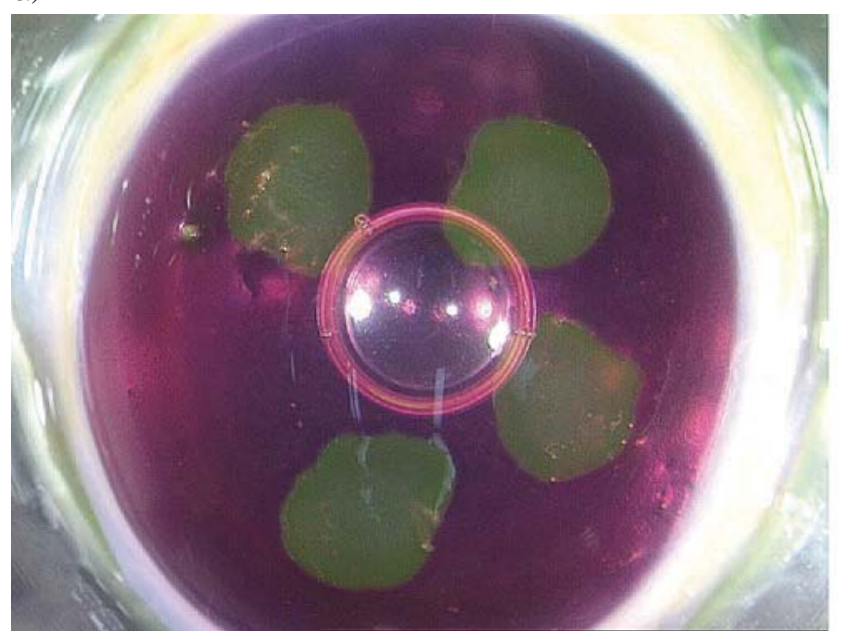

b)

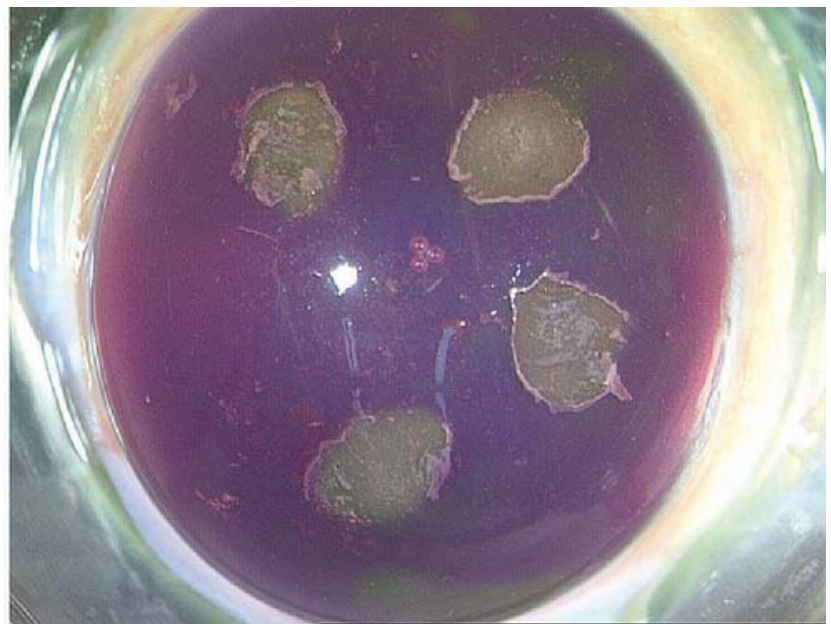

MEM was added at the rate of 1 drop per hour. The images show the isolated cornea a) at the beginning (Day 0) and b) at the end (Day 4) of the experiment.

the end of the experiment. Further increasing the concentration of $\mathrm{BAC}$ to $0.05 \%$ and $0.1 \%$ led to increasingly-worse lesions and partially stripped-off epithelia, with stromal deformation and full necrosis (Figures 5d and 5e).

The correlation of these adverse effects with BAC concentration is clear from Figure 6. At $0.1 \%$, BAC caused the almost immediate destruction of the tissue, and no lactate production was detectable from the third day of the experiment. This, together with the visible degradation of the cornea, indicated the complete death of all cellular material.

\section{Figure 4: A cornea within the EVEIT, treated with hyaluronate citrate drops and artificial tears}

a)

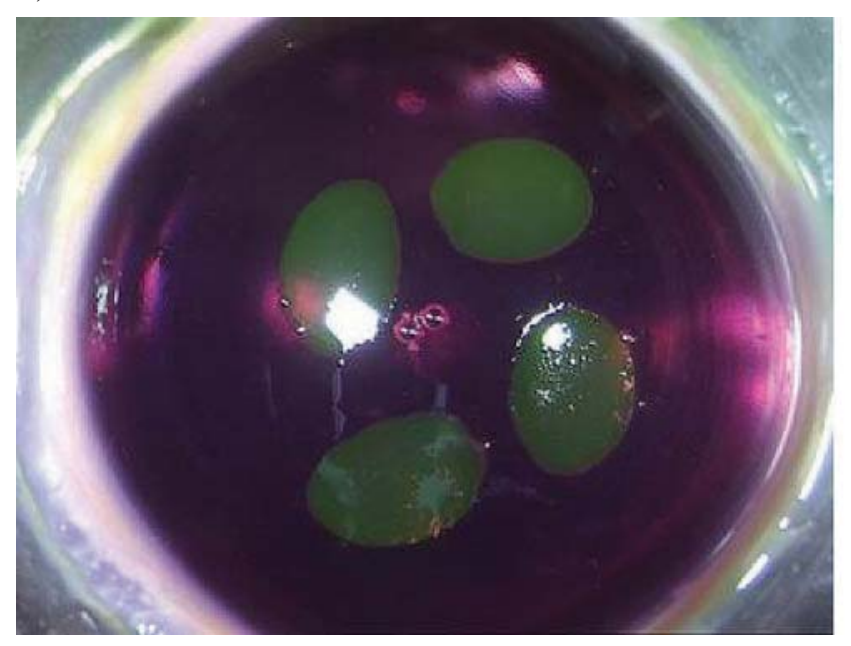

b)

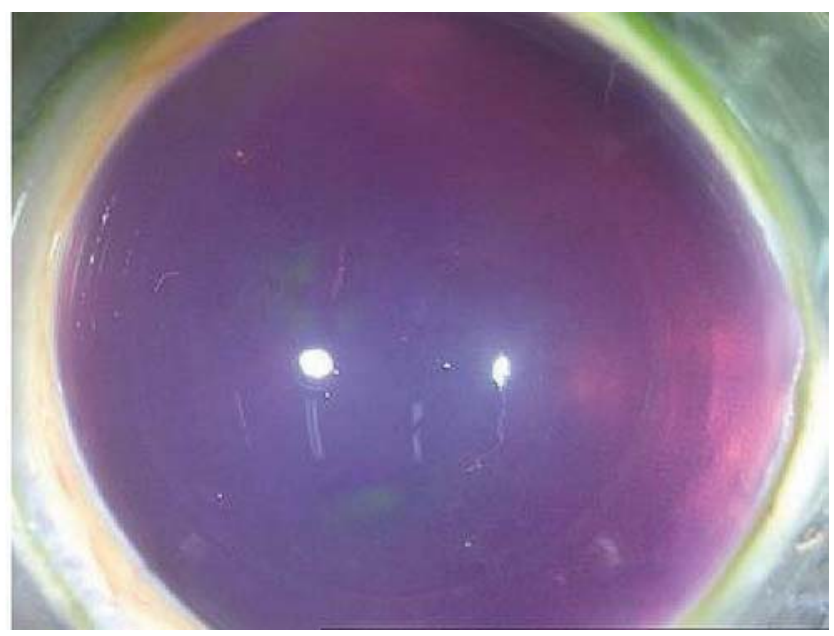

Hyaluronate citrate and artificial tears were added at the rate of 1 drop per hour. The images show the isolated cornea a) at the beginning (Day 0) and b) at the end (Day 4) of the experiment. 
Figure 5: Corneas within the EVEIT, treated with hyaluronate citrate drops and artificial tears containing a range of BAC concentrations

a) $0.001 \% \mathrm{BAC}$

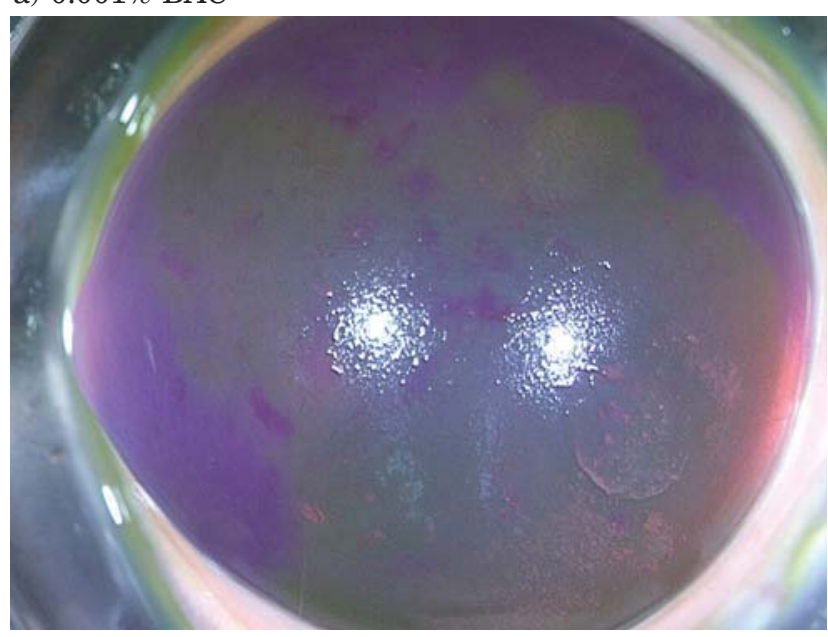

c) $0.025 \% \mathrm{BAC}$

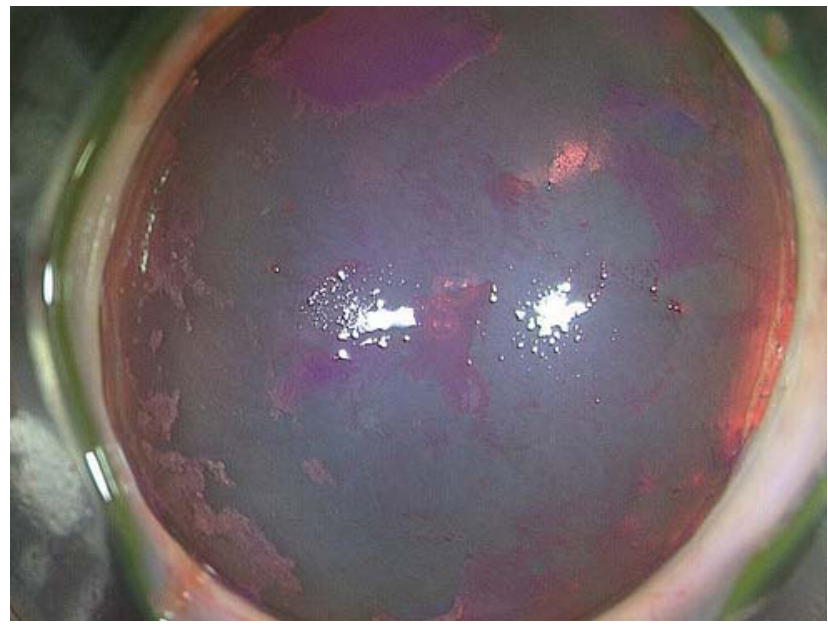

b) $0.01 \% \mathrm{BAC}$

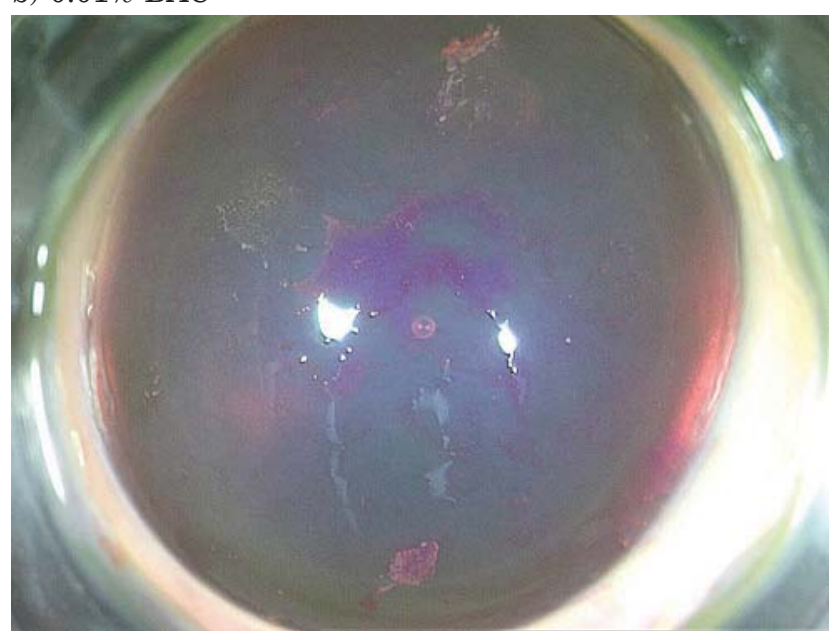

d) $0.05 \% \mathrm{BAC}$

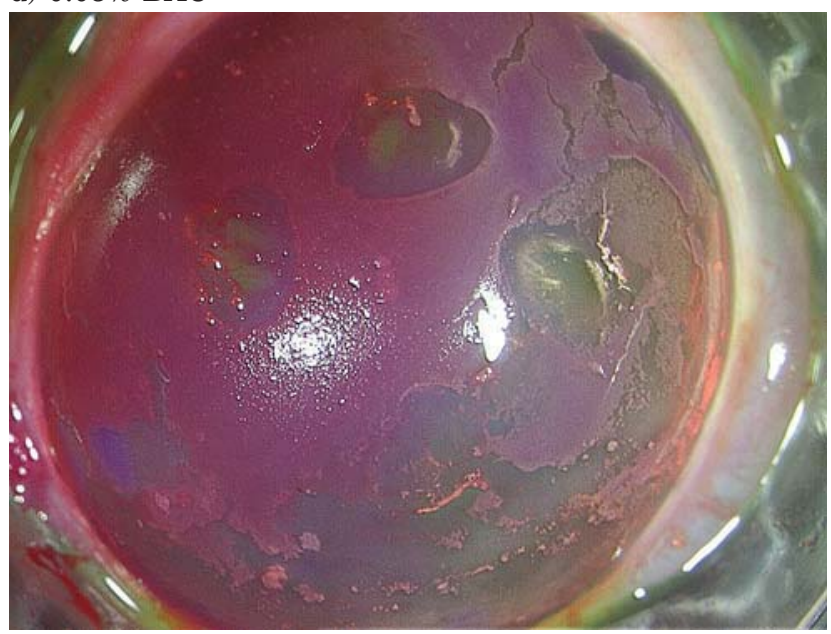

\section{e) $0.1 \% \mathrm{BAC}$}

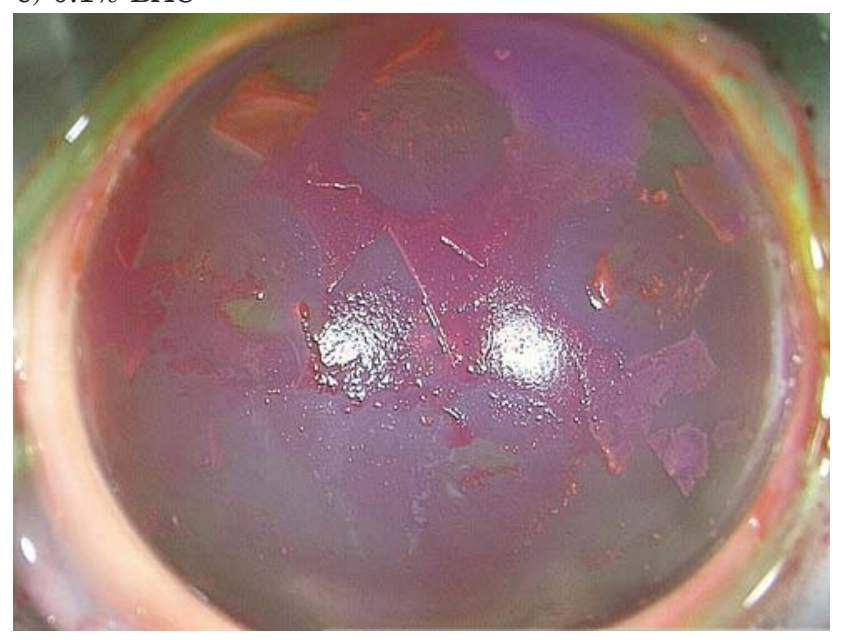

Hyaluronate citrate and artificial tears, with different BAC concentrations, were added at the rate of 1 drop per hour. Photographs were taken after a 4-day treatment. 


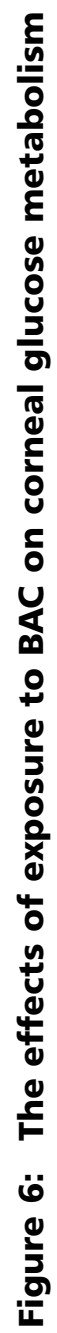
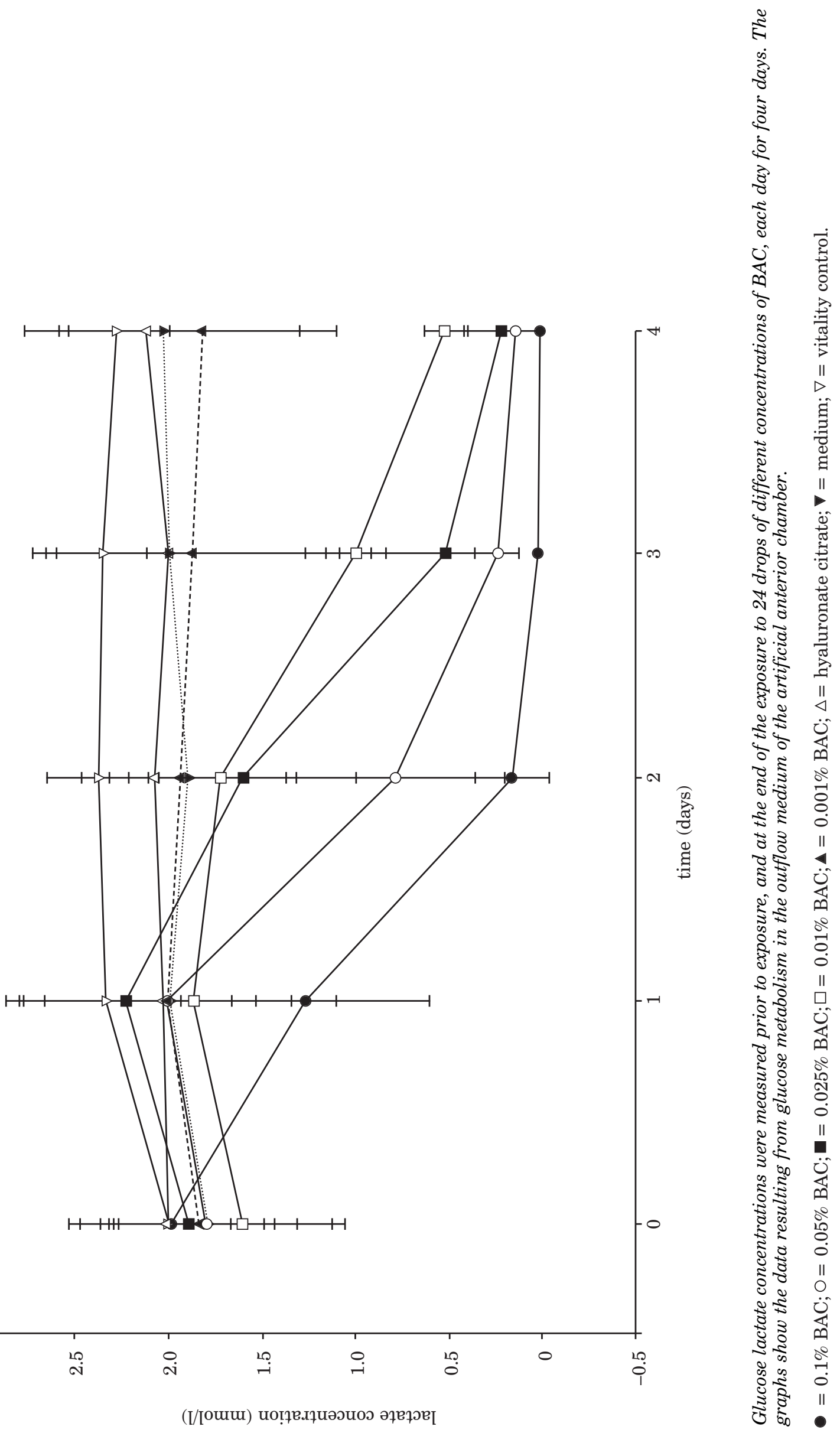


\section{Discussion}

Previous studies have confirmed that BAC in eye drops improves penetration (7), is toxic upon single application (8), more toxic upon multiple applications (9), and may cause damage at very low concentrations (10), at least to the epithelial layer of the cornea (9). The results from the present study confirm the morphological changes that have been previously observed by others upon fluoresceinstaining of the cornea in humans and in rats to determine morphological damage $(9,10)$. Imayasu et al. (11) reported severe damage to rabbit eyes from BAC exposure, reflected by lactate dehydrogenase (LDH) release from corneas in vivo. This correlates with the results from our lactate determinations, which, in contrast to the use of cytosolic LDH release as the classic indicator of membrane defects, directly reflect the breakdown of metabolic activity within the cells. LDH as marker of cellular integrity, and lactate as marker of cellular metabolic activity, are closely related to the vitality of the corneal tissue. The dose-dependent impairment of lactate production during repeated BAC exposure is a clear and simple indicator of cellular damage, which is less susceptible to measurement errors than is the LDH assay.

There is clear evidence that the results obtained with the EVEIT model, presented here in the specific case of BAC exposure, correlated well with the morphological findings observed in humans (9), in all the types of experimental animal cell lines studied (12), and with excised isolated corneas from goat, sheep and buffalo (7). Multiple exposures over several days, with the possibility of surviving tissue and ongoing damage assessment, are the exceptional advantages of our method that have so far only been achieved with live animals. By specific determination of the extent of healing after corneal mechanical trauma, during the concurrent repeated exposure to BAC and non-irritants, we were able to demonstrate that the EVEIT is a good predictor of the dose-dependent toxicity in this specific case. Further experiments are soon to be published, to confirm the suitability of the EVEIT model for the testing of other chemicals.

We observed different stages of corneal healing after different types of repeated application, and a direct effect on the vitality status of the cornea. The EVEIT system showed that after deliberate damage, citrate-buffered hyaluronate drops improved epithelial healing, with a constant rate of epithelial closure, even with larger corneal defects. The EVEIT was able to identify the toxic effects of different concentrations of BAC, in a model that is both time-efficient and, more importantly, does not require the use of animals specifically obtained for this purpose. This replacement of animals by the EVEIT was fundamental to the achievement of one of the major targets of the project, that it should support the principles of the Three Rs (reduction, refinement and replacement).

We are convinced that the EVEIT is a promising candidate for replacing the Draize test in pharmaceutical research on the cornea, and that it could be used as a possible eye-irritation test within the framework of the REACH initiative on chemicals.

\section{Acknowledgements}

This work was partially supported by Ursapharm Arzneimittel GmbH \& Co. KG, Saarbrücken, Germany, who supplied the hyaluronate eye drops containing citrate. Staff costs, equipment, and other materials were financed by ACTO e.V., and by the Department of Ophthalmology, University Hospital, Aachen, Germany. Ursapharm GmbH had no influence on the proposed working programme, or on the results, data interpretation or text presented here.

Received 26.03.07; received in final form 07.09.07; accepted for publication 17.09.07.

\section{References}

1. Draize, J.H., Woodward, G. \& Calvery, H.O. (1944). Methods for study of irritation and toxicity of substances applied topically to the skin and mucous membranes. Journal of Pharmacology \& Experimental Therapeutics 82, 377-390.

2. ECETOC (1998). ECETOC Technical Report No. 48, Eye Irritation: Reference Chemicals Data Bank, 2nd edn, 236pp. Brussels, Belgium: ECETOC.

3. Prospisil, H. \& Holzhuetter, H.G. (2001). A compartment model to calculate time dependent concentration profiles of topically applied chemical compounds in the anterior compartments of the rabbit eye. ATLA 29, 347-365.

4. Van Goethem, F., Adriaens, E., Alépée, N., Straube, F., De Wever, B., Cappadoro, M., Catoire, S., Hansen, E., Wolf, A. \& Vanparys, P. (2006). Prevalidation of a new in vitro reconstituted human cornea model to assess the eye irritating potential of chemicals. Toxicology in Vitro 20, 1-17.

5. Schwab, I.R., Johnson, N.T. \& Harkin, D.G. (2006). Evaluation of inherent risks associated with bioengineered tissue. A case study of protocols for ex vivo expansion and grafting of ocular surface tissue (ARVO 2006 Meeting Abstract). Investigative Ophthalmology \& Visual Science 47, 3942.

6. Redbrake, C., Salla, S. \& Frantz, A. (1999). Metabolic changes of the human donor cornea during organ-culture. Acta Ophthalmologica Scandinavica 77, 266-272.

7. Rathore, M.S. \& Majumdar, D.K. (2006). Effect of formulation factors on in vitro transcorneal permeation of gatifloxacin from aqueous drops. AAPS PharmSciTech 7, Article 57.

8. Labbe, A., Pauly, A., Liang, H., Brignole-Baudouin, F., Martin, C., Warnet, J.M. \& Baudouin, C. (2006). Comparison of toxicological profiles of benzalkonium 
chloride and polyquaternium-1: An experimental study. Journal of Ocular Pharmacology \& Therapeutics 22, 267-278.

9. Ishibashi, T., Yokoi, N. \& Kinoshita, S. (2003). Comparison of the short-term effects on the human corneal surface of topical timolol maleate with and without benzalkonium chloride. Journal of Glaucoma 12, 486-490.

10. Whitson, J.T., Cavanagh, H.D., Lakshman, N. \& Petroll, W.M. (2006). Assessment of corneal epithelial integrity after acute exposure to ocular hypotensive agents preserved with and without benzalkonium chloride. Advances in Therapy 23, 663-671.
11. Imayasu, M., Moriyama, T., Ohashi, J., Ichijima, H. \& Cavanagh, H.D. (1992). A quantitative method for $\mathrm{LDH}, \mathrm{MDH}$ and albumin levels in tears with ocular surface toxicity scored by Draize criteria in rabbit eyes. The CLAO Journal 18, 260-266.

12. Huhtala, A., Alajuuma, P., Burgalassi, S., Chetoni, P., Diehl, H., Engelke, M., Marselos, M., Monti, D., Pappas, P., Saettone, M.F., Salminen, L., Sotiropoulou, M., Tahti, H., Uusitalo, H. \& Zorn-Kruppa, M. (2003). A collaborative evaluation of the cytotoxicity of two surfactants by using the human corneal epithelial cell line and the WST-1 test. Journal of Ocular Pharmacology \& Therapeutics 19, 11-21. 